\title{
The effect of the isotope on the H-mode density limit
}

A. Huber ${ }^{1}$, S. Wiesen ${ }^{1}$, M. Bernert ${ }^{2}$, S. Brezinsek ${ }^{1}$, A. V. Chankin ${ }^{2}$, G. Sergienko ${ }^{1}$, V. Huber ${ }^{3}$,

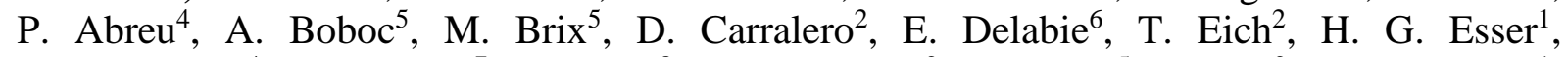
C. Guillemaut ${ }^{4}$, S. Jachmich ${ }^{7}$, E. Joffrin ${ }^{8}$, A. Kallenbach ${ }^{2}$, U. Kruezi ${ }^{5}$, P. Lang ${ }^{2}$, Ch. Linsmeier ${ }^{1}$, C. G. Lowry ${ }^{9,10}$, C. F. Maggi ${ }^{5}$, G. F. Matthews ${ }^{5}$, A. G. Meigs ${ }^{5}$, Ph. Mertens ${ }^{1}$, F. Reimold ${ }^{1}$, J. Schweinzer ${ }^{2}$, G. Sips ${ }^{9,10}$, M. Stamp ${ }^{5}$, E. Viezzer ${ }^{2}$, M. Wischmeier ${ }^{2}$, H. Zohm², JET contributors* and ASDEX Upgrade Team

EUROfusion Consortium, JET, Culham Science Centre, Abingdon, OX14 3DB, UK

${ }^{1}$ Forschungszentrum Jülich GmbH, Institut für Energie- und Klimaforschung - Plasmaphysik, Partner of the Trilateral Euregio Cluster (TEC), 52425 Jülich, Germany

${ }^{2}$ Max-Planck-Institut für Plasmaphysik, D-85748 Garching, Germany

${ }^{3}$ Forschungszentrum Jülich GmbH, Supercomputing Centre, 52425 Jülich, Germany

${ }^{4}$ Instituto de Plasmas e Fusão Nuclear, Instituto Superior Técnico, Universidade Lisboa, PT

${ }^{5}$ CCFE, Culham Science Centre, Abingdon, OX14 3DB, UK

${ }^{6}$ Oak Ridge National Laboratory, Oak Ridge, Tennessee, USA

${ }^{7}$ Laboratory for Plasma Physics, ERM/KMS, B-1000 Brussels, Belgium

${ }^{8}$ CEA, IRFM, F-13108 St Paul-lez-Durance, France

${ }^{9}$ European Commission, B1049 Brussels, Belgium

${ }^{10}$ JET Exploitation Unit, Culham Science Centre, Abingdon OX14 3DB, UK

*See the author list of "Overview of the JET results in support to ITER" by X. Litaudon et al. to be published in Nuclear Fusion Special issue: overview and summary reports from the 26th Fusion Energy Conference (Kyoto, Japan, 17-22 October 2016)

\section{E-mail contact of main author: A.Huber@fz-juelich.de}

Abstract. In order to understand the mechanisms for the H-mode density limit in machines with fully metallic walls, systematic investigations of $\mathrm{H}$-mode density limit plasmas in experiments with deuterium and hydrogen external gas fuelling have been performed on JET-ILW.

The observed H-mode density limit on JET in D- as well as in H-plasmas demonstrates similar operation phases: the stable H-mode phase, degrading H-mode, breakdown of the H-mode with energy confinement deterioration accompanied by a dithering cycling phase, followed by the L-mode phase.

The density limit is not related to an inward collapse of the hot core plasma due to an overcooling of the plasma periphery by radiation. Indeed, independently of the isotopic effect, the total radiated power stay almost constant during the H-mode phase until the H-L back transition. It was observed in D- and H-plasmas that neither detachment, nor the X-point MARFE itself do trigger the H-L transition and that they thus do not present a limit on the plasma density. It is the plasma confinement, most likely determined by edge parameters, which is ultimately responsible for the H-mode DL.

By comparing similar discharges but fuelled with either deuterium or hydrogen, we have found that the H-mode density limit exhibits a dependence on the isotope mass: the density limit is up to $35 \%$ lower in hydrogen compared to similar deuterium plasma conditions (the obtained density limit is in agreement with the Greenwald limit for D-plasma). In addition, the density limit is nearly independent of the applied power both in deuterium or hydrogen fuelling conditions.

The measured Greenwald fractions are consistent with the predictions from a theoretical model based on an MHD instability theory in the near-SOL. The JET operational domains are significantly broadened when increasing the plasma effective mass (e.g. tritium or deuteriumtritium operation), i.e. the $\mathrm{L}$ to $\mathrm{H}$ power threshold is reduced whereas the density limit for the L-mode back transition is increased.

Keywords: density limit, H-mode, detachment, Greenwald fraction, JET 


\section{Introduction}

Tokamak operation at high density with a partially or fully detached divertor is considered as the baseline scenario for ITER [1], DEMO [2] and future fusion power plants. The establishment of a detached divertor at densities close to the Greenwald limit $\mathrm{n}_{\mathrm{GW}}$ [3] is mandatory for maximising the fusion power and for successful operation of future reactors to reduce the heat loads on plasma-facing components, in particular on the divertor target plates, to an acceptable level and to reduce the tungsten sputtering. Despite substantial efforts to quantify the density limit in machines with fully metallic walls, such as JET and AUG tungsten [4], not all underlying mechanisms responsible for the H-L transition are fully understood yet. In particular, the influence of isotopic effects can lead to a significant impact on the density limit (DL).

In this contribution, we investigate the isotopic effect on H-mode density limit in the fully metallic machine JET-ILW. Additionally, the work is extended to compare the H-mode density limits measured in various experimental conditions normalised to the Greenwald density with the predicted values derived from a heuristic model based on the SOL pressure threshold of an MHD instability as recently proposed by Goldston [5].

\section{Experiments}

\subsection{H-Mode density limit Experiment in the JET Tokamak with ITER-like wall}

To investigate the influence of isotopic effects on the DL, a series of experiments was started at JET in D- (with plasma currents $\mathrm{I}_{\mathrm{p}}=1.75-2.5 \mathrm{MA}$ and toroidal magnetic fields $\mathrm{B}_{\mathrm{T}} \approx 1.8-3.4 \mathrm{~T}$ corresponding to $q 95$ in the range 2.8 to 5.9$)$ and in $\mathrm{H}$-plasmas $\left(\mathrm{I}_{\mathrm{p}}=0.9-2.0 \mathrm{MA}\right.$ and $\mathrm{B}_{\mathrm{T}} \approx 1.3-1.8 \mathrm{~T}$ corresponding to $q 95$ in the range 2.8 to 6.0 ) at varying levels of input power in the low triangularity vertical target configuration with high deuterium/protium fuelling.

This H-mode density limit (H-mode DL) constitutes an effective undisruptive density limit, a so-called 'soft limit', as it is a back transition to L-mode and the plasma operation can be continued with a lower confinement. For the studies of the DL, gas ramp-up discharges were conducted to achieve the maximum densities necessary for the H-L back transition. Fig.1 shows the time evolution of a typical H-mode density limit discharge in JET-ILW in low-triangularity magnetic equilibria (average triangularity of $\delta=0.22$ ). The gas ramp-up is realized by external gas fuelling of neutral gas into the inner leg of the divertor with a rate of up to $2 \times 10^{23} \mathrm{D} / \mathrm{s}$ to raise the plasma density up to the DL. Auxiliary neutral beam heating of $\mathrm{P}_{\mathrm{NBI}}=10 \mathrm{MW}$ ensures 
that the discharges are well above the L-H power threshold of about 3.0-4.0 MW. The neutral pressure during the DL studies increases up to $2.0 \mathrm{~Pa}$ in the divertor and $0.006 \mathrm{~Pa}$ in the main chamber. The BeII fast emission signal in the outer divertor represents the ELMs behaviour during the density ramp-up. When the density is raised by gas puffing, the confinement

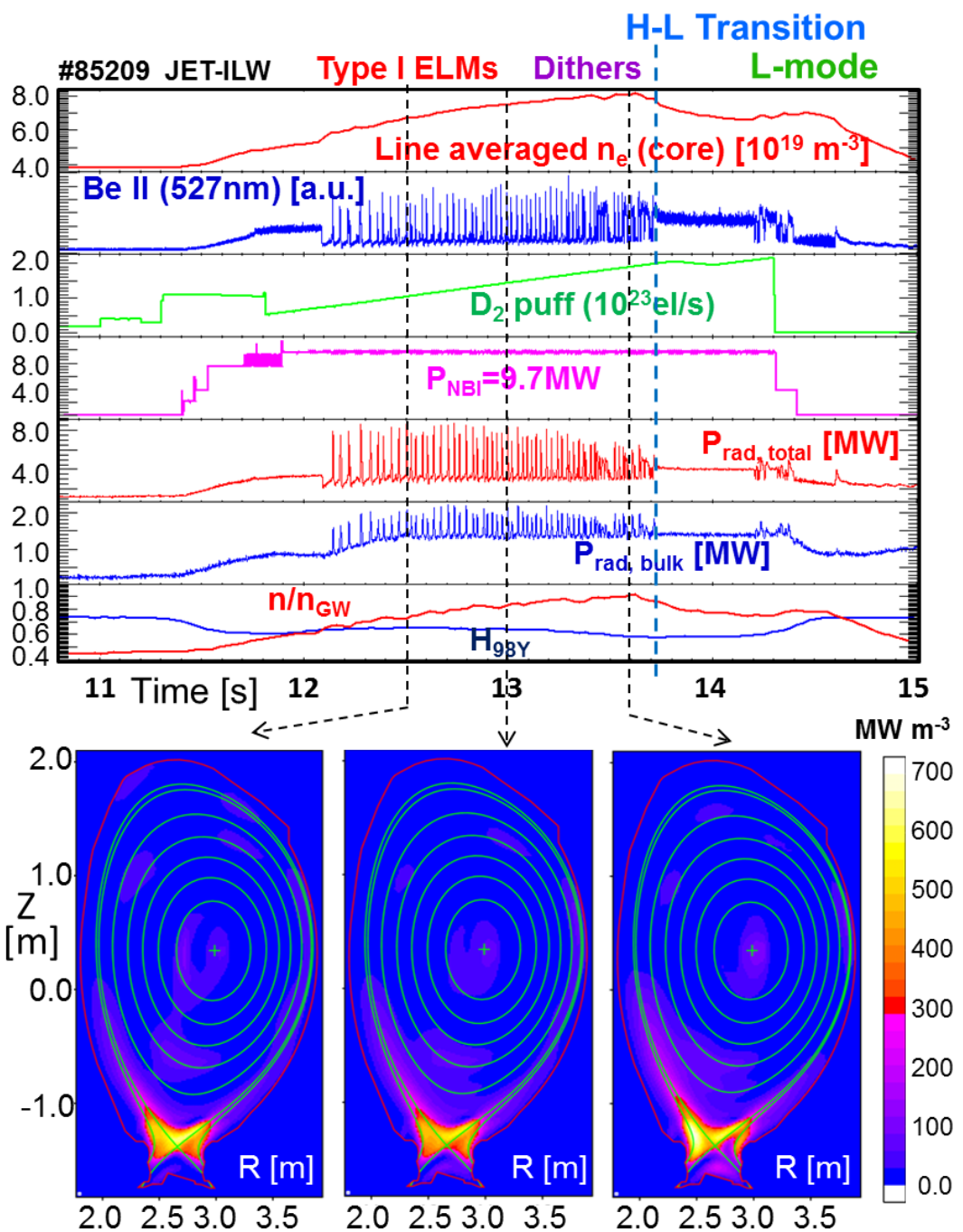

Fig.1 Time evolution of a typical H-mode density limit discharge on JET-ILW. Also shown are the poloidal radiation distributions during different time phases of the H-mode DL discharge. The total as well as the bulk radiation stay constant throughout the discharge.

enhancement factor remains at a constant level of $\mathrm{H} 98 \mathrm{Y}=0.65$ up to about $\mathrm{n}_{\mathrm{e}} / \mathrm{n}_{\mathrm{GW}}=0.9$. Further gas puffing leads to a moderate increase of the density, but the confinement deteriorates strongly down to $\mathrm{H} 98 \mathrm{Y}=0.56$ and the discharges usually make a back transition into the $\mathrm{L}$ mode. Prior to the final transition into L-mode, type I ELMs are replaced by a sequence of H-L-H transitions, with short periods of H-mode embedded in the otherwise L-mode phase of the discharge, as it will be discussed in detail in section 3.2. The sequence of these transitions is 
what we will call 'dithering H-mode'. The dithering cycles can be seen as a modulation of the BeII signal in the divertor.

The energy losses due to radiation can cool down the confined plasma and thus cause the $\mathrm{H}$ mode density limit. In previous studies at ASDEX Upgrade (AUG) and JET, both with a fully carbon covered wall, the density limit of H-mode has been attributed to the full detachment of the divertor [6] with a significant role of the radiation. However, in the JET-ILW as well as in full-W ASDEX the poloidal radiation distribution as shown in Fig.1 for a JET H-mode DL pulse and the total radiated power as well as the radiation power in the main chamber $\left(\mathrm{P}_{\text {rad,bulk }}\right)$ stay almost constant during the H-mode phase until the H-L transition. Additionally, the

radiation fraction, $\gamma_{\text {rad }}=\frac{P_{\text {rad }}}{P_{\text {heat }}}$, is low and is about $0.3(0.4-0.6$ for AUG). Therefore, the density limit is not related to additional energy losses from the confined region by radiation. It is also not related to inward collapse of the hot discharge core induced by overcooling of the plasma periphery by radiation. The effective ion charge, $Z_{\text {eff, }}$ is $\approx 1.15$ in the density ramp-up pulses. Replacement of the first wall and divertor walls by metallic materials reduced significantly the carbon levels [8], resulting in a reduced radiation and reduced $Z_{\text {eff. }}$ Under cold detached divertor conditions with significant reduction of $\mathrm{W}$ sputtering, the tungsten is introduced into the plasma only during the ELMs. Beryllium is a much weaker radiator than carbon. A significant fraction of the radiation is caused by deuterium [8]. A MARFE (at the X-point) does not appear before the $\mathrm{H}-\mathrm{L}$ transition and, thus, is not related to the H-mode density limit.

\section{Characterisation of the H-mode density limit}

\subsection{Characterisation of the H-mode DL on JET-ILW}

The evolution of a gas fuelled, high density $\mathrm{H}$ mode discharge in JET-ILW can be described by three main stages as shown in Fig.1: a Type I ELM phase, then a dithering cycling phase with energy confinement deterioration, followed by the L-mode phase. The impact of the phases on the plasma stored energy and the central line averaged electron density is shown in Fig.2.

The characteristics of the phases and their impact on the electron density and temperature profiles (see Fig.2) are listed in the following:

Stable H-mode Before the dithering phase, the stored energy and the confinement stay constant whereas the density is increasing in the core and in the edge. At the same time, the pedestal temperature $\left(\mathrm{T}_{\mathrm{e}, \mathrm{ped}}\right)$ decreases during the gas ramp-up so that the pedestal pressure stays constant. This phase is called a stable H-mode, since the pressure, and thus the confinement, stay constant while the density increases. 


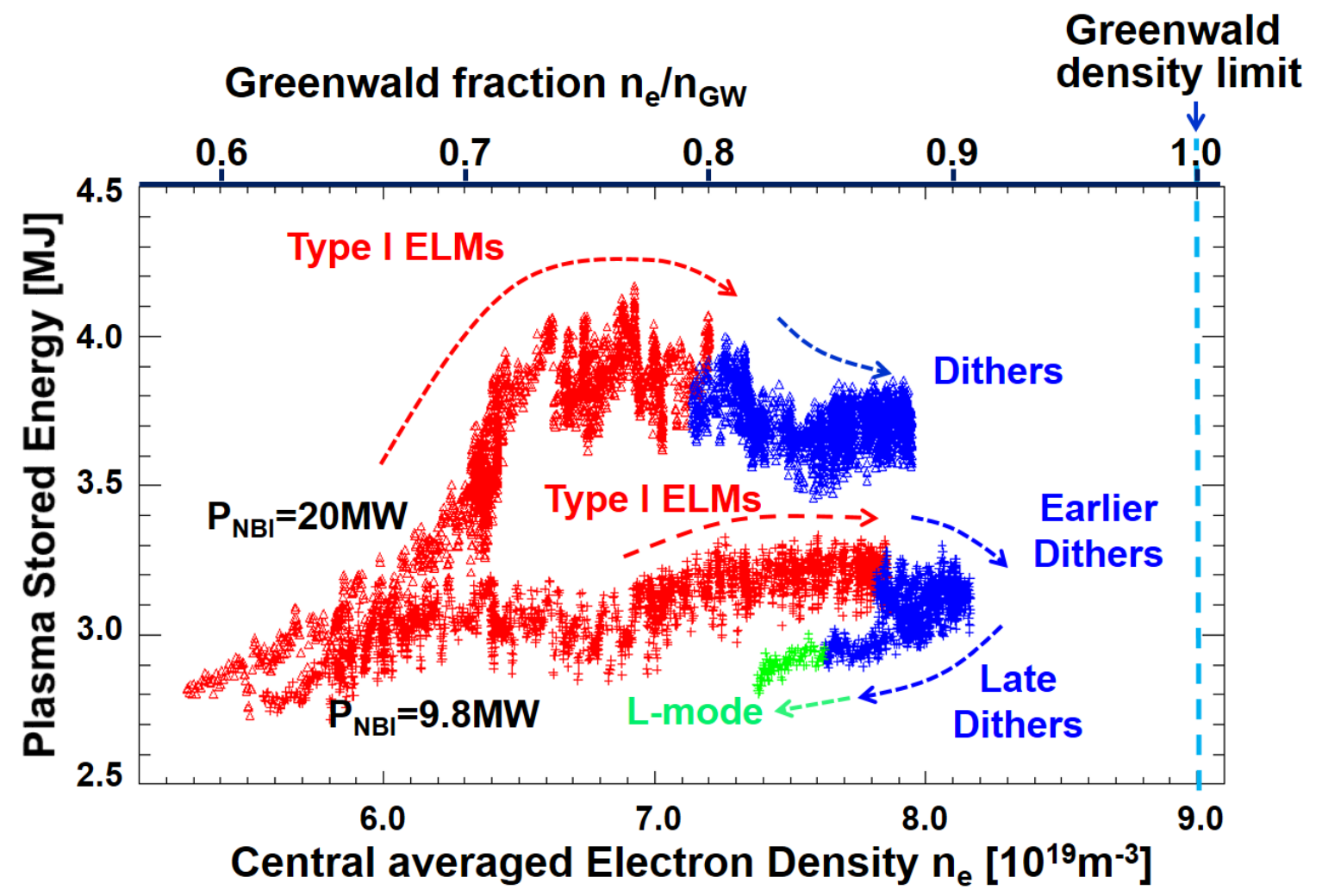

Fig.2 Plasma stored energy plotted versus the central averaged electron density. The main phases of the H-mode DL on JET-ILW. Picture adapted from [7].

Dithering cycling phase Two distinct sub-phases are identified in the 'dithering phase': an early 'dithering phase' with confinement degradation and a late phase with the breakdown of the H-mode.

Earlier dithering phase During the earlier 'dithering phase', the density increases marginally and $\mathrm{T}_{\mathrm{e}, \mathrm{ped}}$ cools down, degrading the pedestal pressure due to a reduction of the confinement and of the stored energy.

Late dithering phase Shortly before the $\mathrm{H}-\mathrm{L}$ back transition during the late dithers a strong altering of the stored energy as well as a density drop by $15 \%$ have been observed, followed by the L-mode phase. The duration of the dithering phase varies, between the discharges in the database, from $0.1 \mathrm{~s}$ to over $0.5 \mathrm{~s}$. Systematically the dithering cycling has been observed in the configurations with both strike points on the vertical targets but only occasionally, or with very short duration of the dithering phases, in the configuration with the outer strike point positioned on the horizontal divertor plate. In the latter configuration an increase of the fuelling source is associated with a transition from type I to small ELMs combined with dithering cycles, which finally leads to the back transition.

L-mode In the last phase, the density decreases over the full profile and the temperature further reduces. 
It should be mentioned here that the operation phases of the H-mode DL in AUG are identical to the findings on JET-ILW.

\subsection{Dithering cycles prior to the L-mode}

Independently of the isotopic mass of the main plasma, it has been observed that the transition
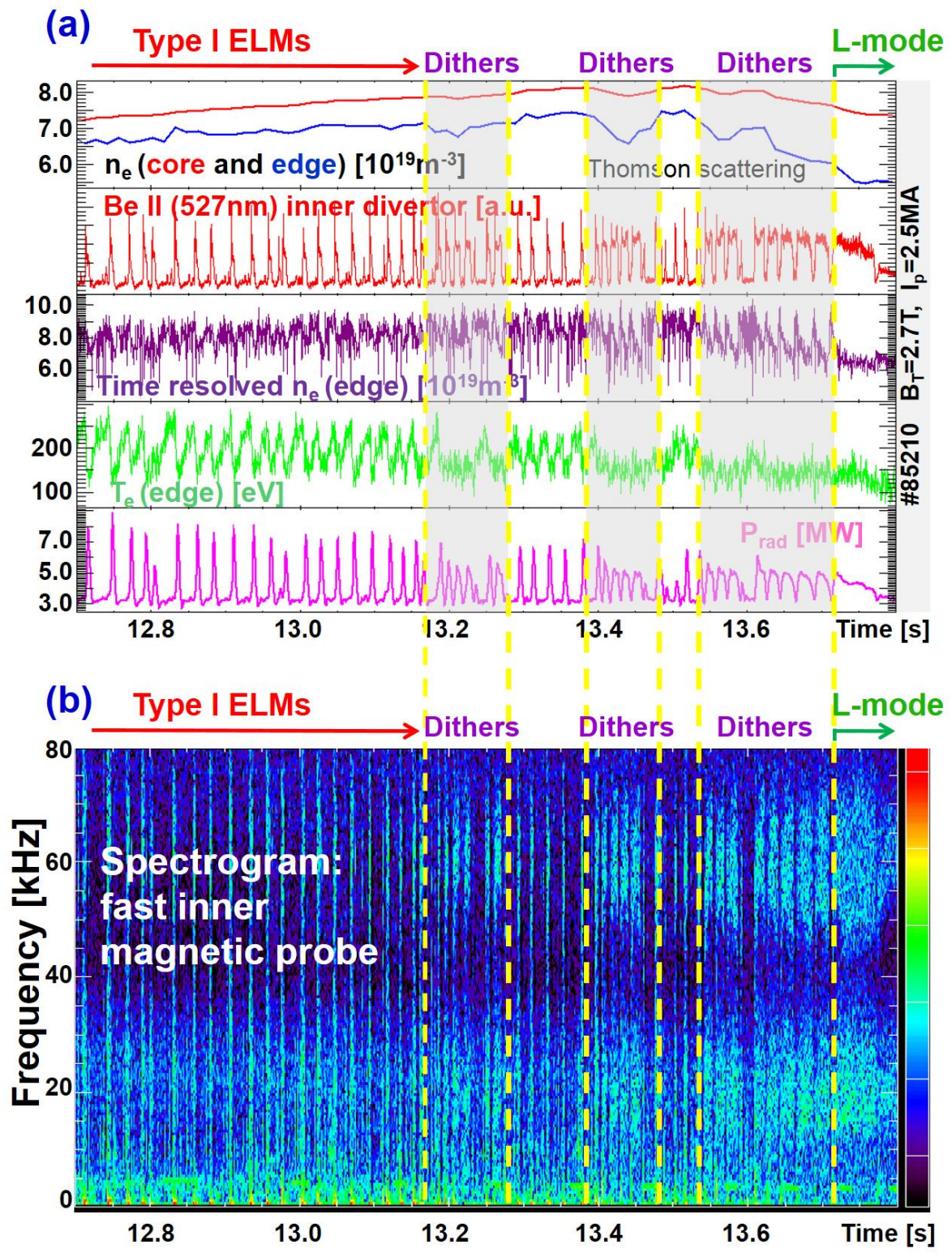

Fig. 3 Characteristic time traces of the high density limit JET-ILW discharges for dithering cycles before the H-L transition: (a) central and edge line-integrated electron density, BeIIemission signal, time resolved pedestal density $\mathrm{n}_{\text {edge }}$ and temperature $\mathrm{T}_{\text {edge, }}$, total radiated power $\mathrm{P}_{\mathrm{rad}}$ as well as (b) power spectrum of a magnetic probe located inside the vessel on the high field side.

from $\mathrm{H}$-mode to L-mode is not always an abrupt event but may exhibit a series of H-L-H transitions ("dithering H-mode"), or a gradual transition (which is orders of magnitude longer 
than the energy confinement time $\tau_{\mathrm{E}}$ ). Fig.3 shows the details of a 'dithering cycling' phase of typical JET H-mode pulses in a magnetic field configuration with both strike points on the vertical divertor targets. The cause of the repetitive $\mathrm{H}-\mathrm{L}-\mathrm{H}$ transitions must be the modulation of the plasma edge density, so that the sharp density rise during short $\mathrm{H}$-mode periods, reflecting a significant improvement in the particle confinement, triggers back H-L. During transient Hmode periods of the dithers, the inner divertor is completely detached and the outer divertor leg is at least partially detached. In spite of that, one observes that the plasma density both at the edge and in the core during the first sub-phase rises, reflecting a significant improvement in the particle confinement; it demonstrates that the detachment itself presents no limit on the plasma density and the H-L transition. The edge electron temperature, measured by the electron cyclotron emission (ECE) diagnostic [9], does not show any excursion during the dithering cycles and stays almost at the lowest value of $\approx 140 \mathrm{eV}$. Thus, a cold and dense pedestal during the H-mode phase increases significantly the collisionality of the plasma pedestal. This is in line with the explanation given in $[10,11]$ that the H-mode DL could be explained from the requirements that, in the edge transport barrier, the radial pressure gradient does not exceed the ballooning stability threshold and the critical plasma collisionality. The duration of the transient L- and H-phases changes during the development of the dithers. The late dithers demonstrate longer L-phases $(\approx 25 \mathrm{~ms})$ than the early ones $(\approx 10 \mathrm{~ms})$ leading to the reduction of the ne even before the $\mathrm{H}-\mathrm{L}$ transition because the short $\mathrm{H}$-mode periods are not able to provide a full recovery of the density. During the L-phases with the low edge density, the BeII-emission has large values whereas it decreases during the short $\mathrm{H}$-mode phase together with the total radiation. The measurements with fast magnetic probe $(\partial \mathrm{B} / \partial \mathrm{t})$ show that the turbulence level is significantly increased (see Fig.3b) when the L-phase develops during the dithers. Similar to those after the final transition into the L-mode, the broadband fluctuations, reflecting the significant anomalous transport, have been observed in the low confinement dithering phase. As was demonstrated in $[4,12]$, during the dithering cycles the MARFE itself does not drive the H-L transition.

Dithering cycles have not been observed in the majority of the H-mode DL pulses on ASDEX Upgrade or, to be exact, only one DL pulse shows the dithering cycles. In contrary to AUG, on JET-ILW the density after H-L transition shows a strong reduction pushing the plasma again into the H-Mode operational space with the consequent improvement in the particle confinement. This leads subsequently to the increase of the density with the following H-L 
transition. On ASDEX Upgrade, however, the density increases after the H-L transition, driving the plasma away from the H-mode operational space.

\section{Role of Detachment in H-mode density limit}

The H-mode density limits in earlier experiments on JET-C demonstrate a good agreement between experiment and the model developed by Borrass et al. [13]. According to this model,

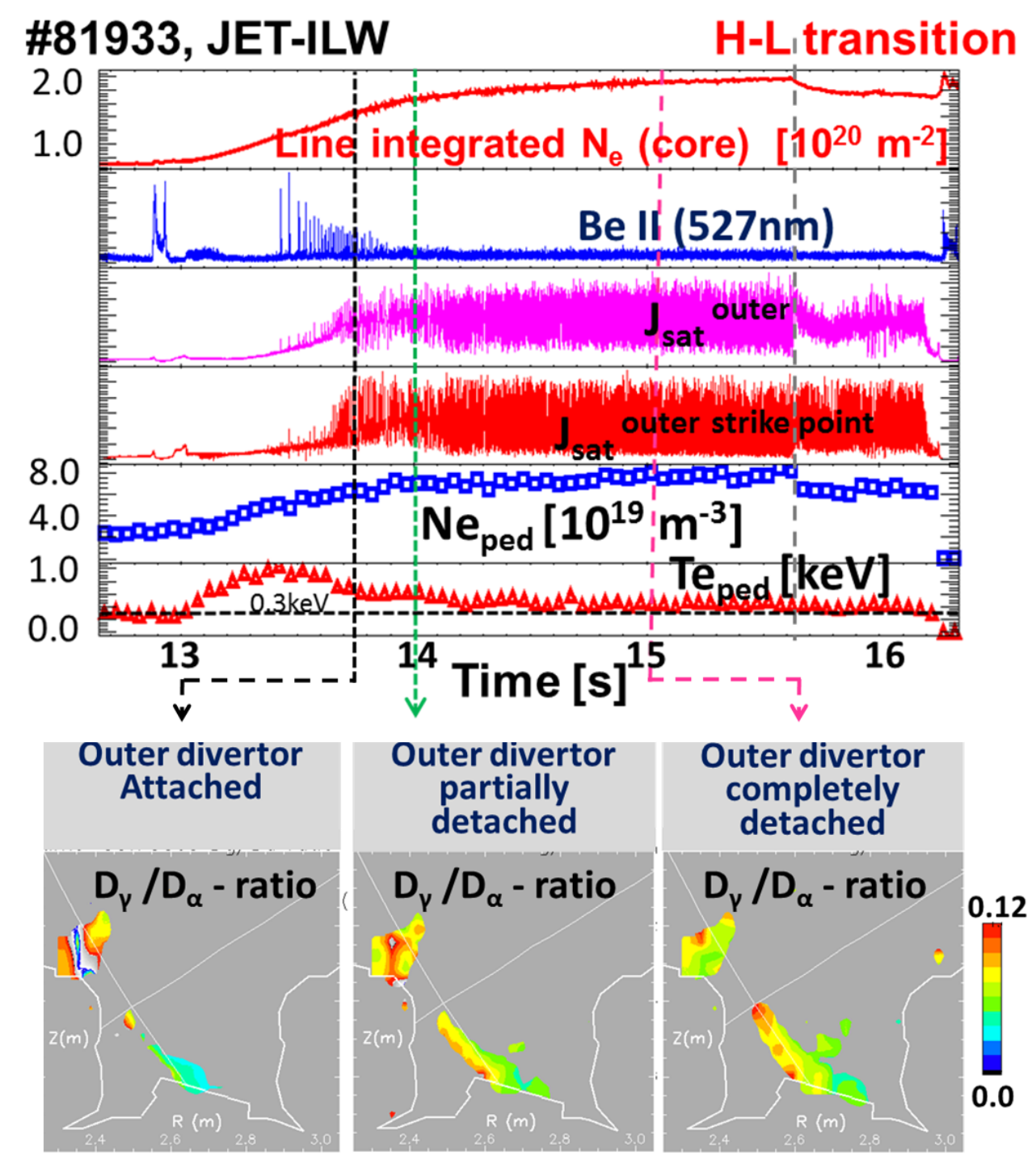

Fig.4 Tomographic reconstructions of $\mathrm{D}_{\gamma} / \mathrm{D}_{\alpha}$ ratio (bottom) in the divertor region during three phases of the H-mode density limit discharge.

the onset of the H-mode density limit is related to the plasma detachment at the divertor plates between ELMs and the H-mode DL can be described by the following empirical scaling [13]: 


$$
n_{H D L}=48.2 \frac{q_{\perp}^{0.049} B_{T}{ }^{0.57} \delta_{a v}^{0.11}}{q_{95}^{0.86} R^{1.07}}
$$

Here, $n_{H D L}$ is the line averaged density at the limit in $10^{19} \mathrm{~m}^{-3}, q_{\perp}$ the mean power flux density across the separatrix surface in $\mathrm{MW} / \mathrm{m}^{2}, B_{T}$ the toroidal magnetic field at the geometric plasma centre in $\mathrm{T}, \delta_{a v}$ the average of upper and lower triangularity, $q_{95}$ the safety factor at the $95 \%$ flux surface and $R$ the major radius of the plasma in $\mathrm{m}$.

The H-mode density limit in the machine with the ILW shows larger values $(>20 \%)$ than predicted by Borrass, indicating in this latter case that the H-L transition cannot be explained by the detachment. Fig. 4 shows the observations made in the divertor of JET-ILW during the approach to the limit in the H-mode density limit discharge. The time traces show measurements of the pedestal density $\mathrm{n}_{\text {ped }}$ and pedestal temperature $\mathrm{T}_{\text {ped. }}$. As well as the integrated ion saturation current to the outer divertor and the local saturation current at the outer strike point. For characterisation of the plasma detachment processes, it is very important to determine the source of impurities and of their radiation distribution in front of all plasmafacing components as well as to determine which regions of the divertor are affected by recombination. A new endoscope [14], having an improved spatial resolution $(\leq 3 \mathrm{~mm}$ in the object plane) over previous designs and delivering images of high quality, gives a unique opportunity for monitoring the impurity radiation as well as the changes in the recombination region by simultaneous imaging of $\mathrm{D}_{\alpha}, \mathrm{D}_{\beta}, \mathrm{D}_{\gamma}$ emission from the divertor with CCD cameras
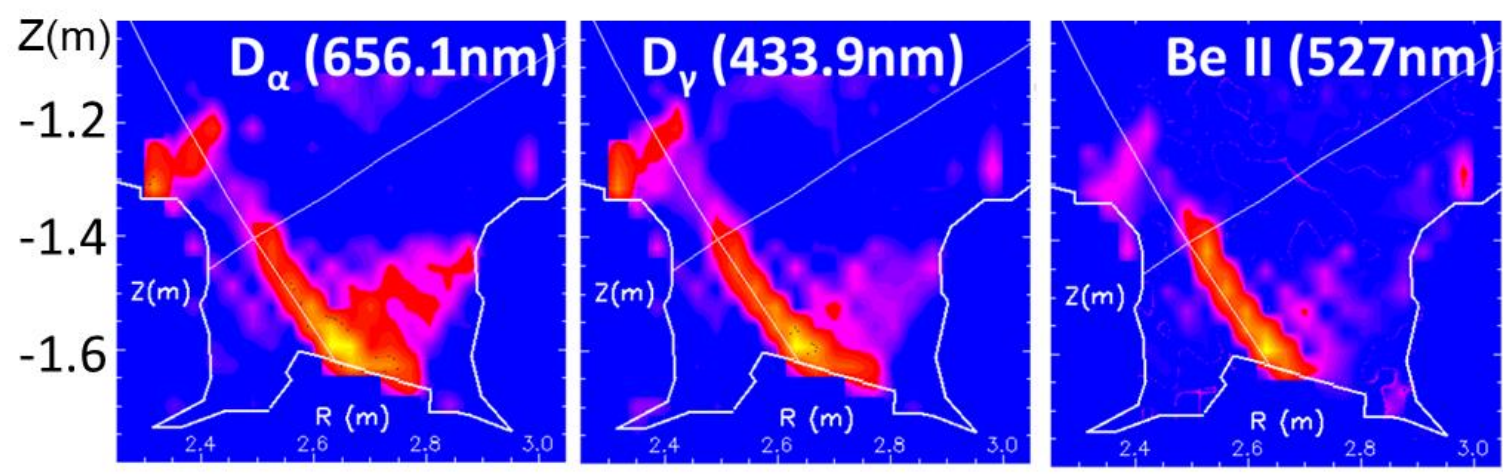

Fig.5 Tomographic reconstructions of $\mathrm{D}_{\alpha^{-}}, \mathrm{D}_{\gamma^{-}}$and BeII-emissions just before the H-L transition at $\mathrm{t}=15.6 \mathrm{~s}$.

with the same Field of View. The recorded images are inverted by a singular value decomposition method [15,16,17] to provide local emissivities (see Fig. 4 bottom and Fig.5). The lower envelope of the saturation currents indicates the roll-over into detachment during the inter-ELM period in the outer divertor at time $\mathrm{t}=14 \mathrm{~s}$. After $\mathrm{t}=14 \mathrm{~s}$ the outer divertor is at least 
partially detached. At $15 \mathrm{~s}$ the $\mathrm{D}_{\gamma}$-emission rapidly increases in the outer divertor. At the same time, the $\mathrm{D}_{\gamma} / \mathrm{D}_{\alpha}$-ratio increases strongly up to the level of $\approx 0.1$ throughout the outer divertor plasma. From ADAS photon emission coefficients [18] the expected $\mathrm{D}_{\gamma} / \mathrm{D}_{\alpha}$-ratio assuming that the radiation is due to electron impact excitation processes should be below 0.02 for $\mathrm{T}_{\mathrm{e}} \leq 5 \mathrm{eV}$. This is much lower than the measured ratio indicating the strong contribution of recombination processes. The electron density at the strike point has been measured by Langmuir probes and amounts to $\approx 0.7-1.0 \times 10^{20} \mathrm{~m}^{-3}$. At such a density and assuming that the main fraction of radiation is due to recombination, the estimated local $\mathrm{T}_{\mathrm{e}}$ calculated from ADAS photon emission coefficients [18] is about 1.0-1.5eV, which is in good agreement with Fujimoto's collisionalradiative model [19].

Additionally, the ion flux to the outer divertor reduces and reaches at $t=15 \mathrm{~s}$ the degrees of detachment $\mathrm{DoD}^{\text {peak}} \approx 5.0$ and $\mathrm{DoD}^{\mathrm{int}} \approx 3.0$, confirming the statement about the fully detached outer divertor. The degree of detachment (DoD) of a given flux-tube is used in the literature [20] for the quantitative measure of the detachment and is defined as the reduction in ion flux at the target relative to what is expected from upstream quantities. The latter is calculated according to the simple Two-Point-Model (TPM), specifically $=\Phi_{\text {ion }}{ }^{T P M}$ calc $/ \Phi_{\text {ion }}{ }^{\text {meas target }}$ , where $\Phi_{\text {ion }}{ }^{\text {TPM calc }}$ is the target ion flux expected using the TPM and $\Phi_{\text {ion }}{ }^{\text {meas target }}$ is the measured ion flux (peak or integral) at the target.

Thus, after $\mathrm{t}=15 \mathrm{~s}$ the outer divertor is fully detached. Despite the fully detached inner and outer divertor the density in the core and at the edge is raised for a long-time period after the outer divertor is fully detached, until the H-L transition. The radiation patterns of hydrogen emission lines as well as Be emission are located in the outer leg outside the LCFS without any indication of a MARFE formation (see Fig.5). Thus, detachment and subsequent MARFE if any do not trigger the $\mathrm{H}-\mathrm{L}$ transition.

A similar conclusion delivers the observation at ASDEX Upgrade [21]. In full-W AUG, the complete detachment sets in after the H-mode DL and, thus, the detachment does not play a key role in the physics of the $\mathrm{H}-\mathrm{L}$ transition.

\section{Impact of the isotopic effects on the density limit}


Although the operation phases are identical for D- and H-plasmas, the DL shows a strong dependence on the isotopic mass, as shown in Fig.6. The DL is up to 35\% lower in the Hplasma than in the deuterium plasma (Fig.6) and it is well predicted by the Goldston model discussed later. Both plasma discharges have been carried out in a similar vertical target
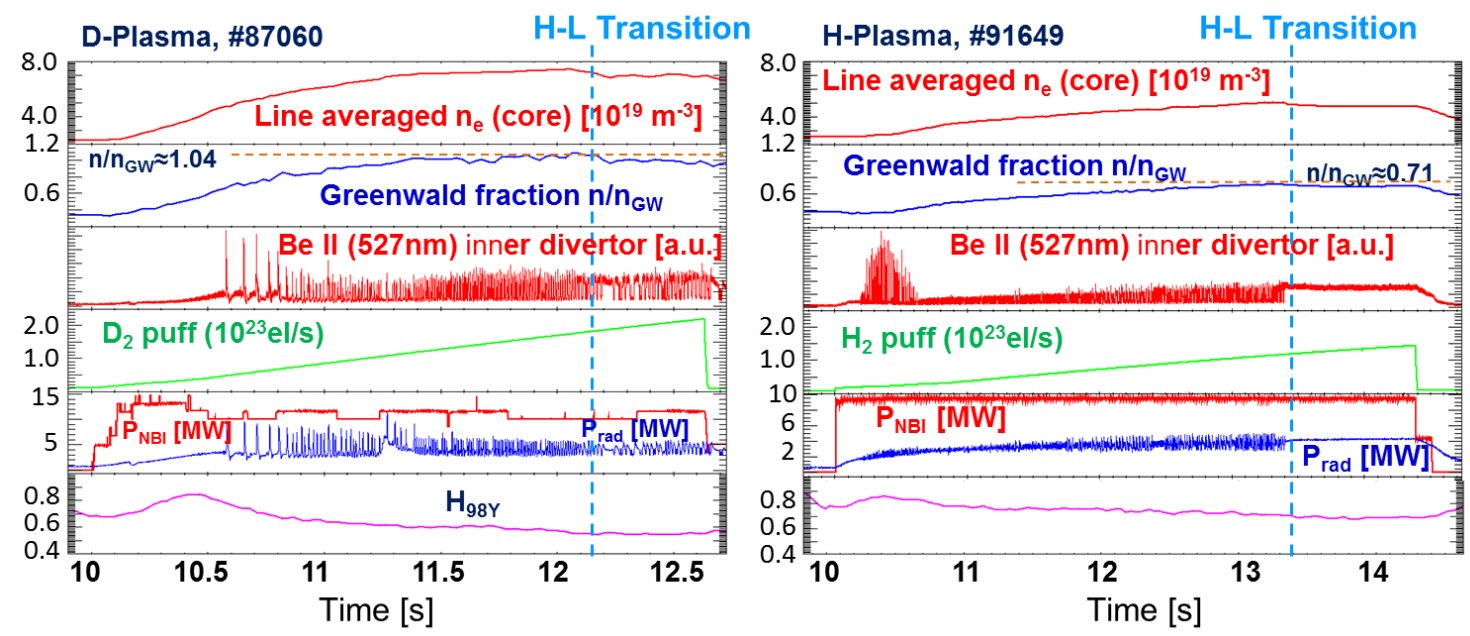

Fig.6 Time traces during a typical density ramp-up experiment on JET-ILW with D-plasma (left) and H-plasma (right). The time when the H-mode DL sets in is marked by the dashed lines.

magnetic field configuration with $\mathrm{I}_{\mathrm{p}} / \mathrm{B}_{\mathrm{T}}=1.9 \mathrm{MA} / 1.8 \mathrm{~T}$ and $\mathrm{q}_{95}=2.8$.
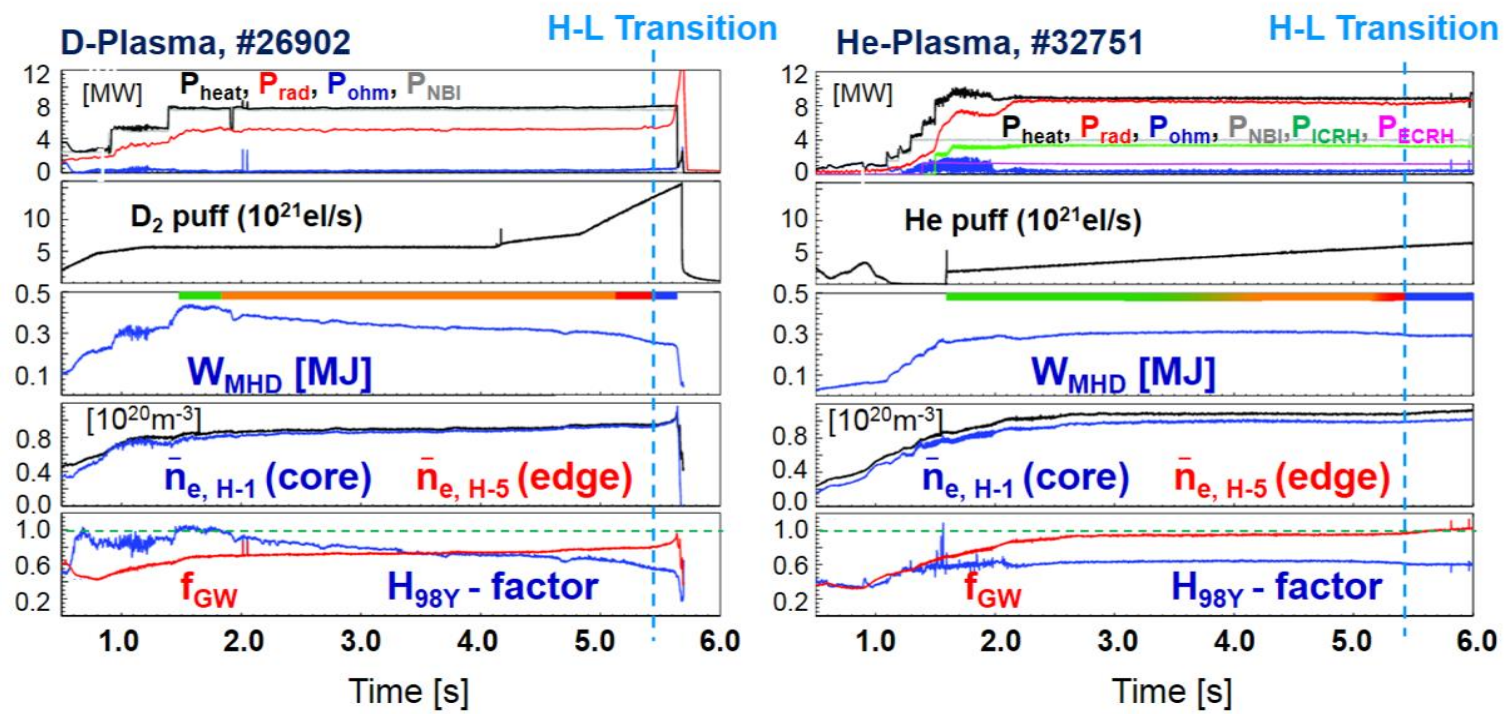

Fig. 7 Time traces of heating powers and radiated power, neutral gas fuelling rate, stored energy, edge and central line averaged electron density, Greenwald fraction and H-factor on AUG with Dplasma (left) and He-plasma (right).

The impact of the atomic mass of the plasma species on the density limit in H-mode pulses has also been observed in AUG with fully metallic wall. The H-mode DL plasma pulses with 
identical main plasma parameters $\left(\mathrm{B}_{\mathrm{T}} / \mathrm{I}_{\mathrm{p}}=2.5 \mathrm{~T} / 0.8 \mathrm{MA}\right)$ and with the same magnetic equilibria have been compared in D- and He-plasmas (see Fig.7). The total heated power in the two pulses is identical and is about $8 \mathrm{MW}$. A deuterium plasma discharge is predominantly a NBI heated discharge. On the other hand, the He-pulse is heated with RF wave heating (ICRH and ECRH) in addition to the neutral beam power. The pulse in He-plasma exhibits an increase in Greenwald fraction by $20 \%\left(f_{\mathrm{GW}}=0.95\right.$ versus 0.79 for deuterium discharge) what is in line with the prediction by the Goldston model.

\section{Impact of the input heat power on the density limit}

The impact of the input heat power on the H-mode density limit at JET in D-plasma has been analysed in the experiments by varying the input heating power. The heating scan does not include a variation of the heating source. Only the intrinsic Ohmic heating and NBI heating were used. A discharge with RF wave heating (ICRH) was not carried out. Fig.8a shows the
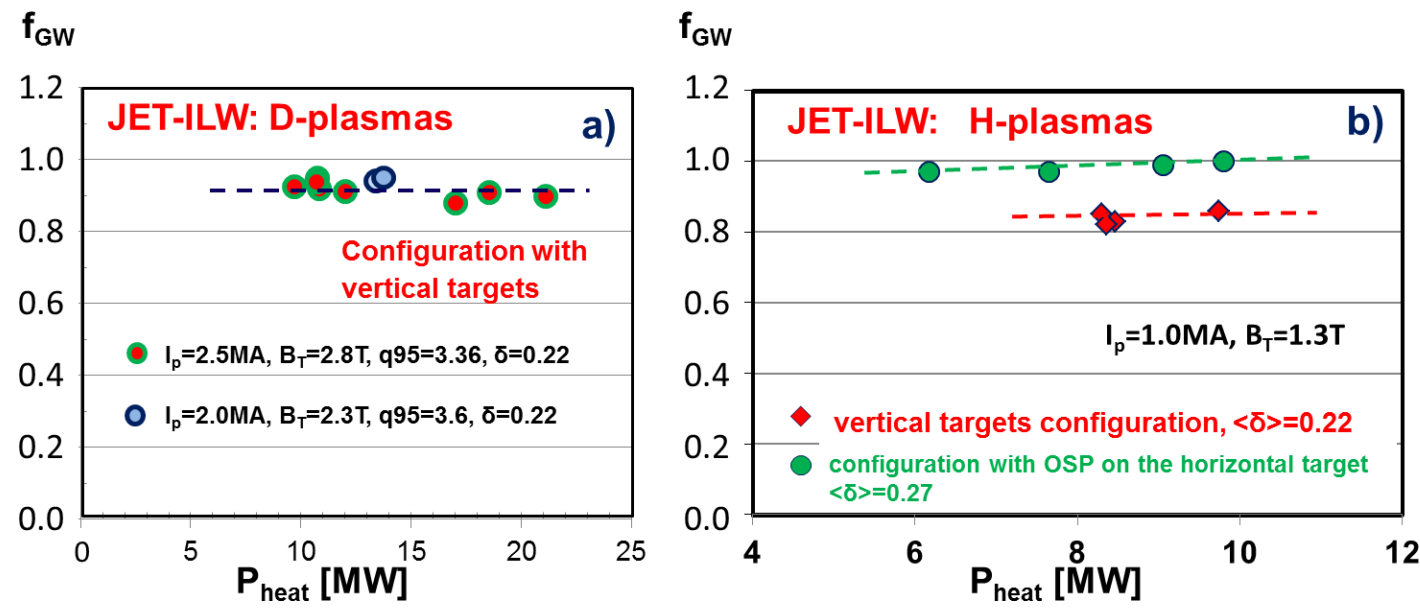

Fig.8 Dependency of the Greenwald fraction at the H-mode DL on the total heating power for JET-ILW in D-plasma (left) and H-plasma (right).

measured Greenwald fractions as a function of the total heating power obtained in these experiments. These D-plasma pulses have been performed in low -triangularity magnetic equilibria at $\mathrm{B}_{\mathrm{T}} \approx 2.7 \mathrm{~T}, \mathrm{I}_{\mathrm{p}}=2.5 \mathrm{MA}, \mathrm{q}_{95}=3.36$, average $\delta=0.22$ with location of the inner and outer strike points on the vertical targets. Basically, the density limit in H mode on JET-ILW is nearly independent of the power at higher heating power beyond 8MW and the corresponding densities only approach in this configuration a Greenwald fraction of about $f_{G W}=0.9$. Nearly the same findings have been observed in H-mode DL experiments with H-plasma. Fig.8b represents the result which was obtained by varying the NBI heating power from $6 \mathrm{MW}$ to $9.4 \mathrm{MW}$ at constant 
plasma current and safety factor for the full-metallic JET in two configurations: vertical target configuration and the configuration with the location of the inner and outer strike points on the vertical target and horizontal target. The achieved densities are nearly constant or show very little increase with the heating power. The density limits reached in the experiments shown in Fig8 $\mathrm{a}$ and $\mathrm{b}$ demonstrate only the lack of dependence on $\mathrm{P}_{\text {heat }}$, the absolute values however cannot be compared with each other because of strong differences in main plasma parameters due primarily to the different plasma currents and magnetic field strengths. The expected $\mathrm{H}$ mode DL in D-plasma at $\mathrm{I}_{\mathrm{p}}=1.0 \mathrm{MA}$ and $\mathrm{B}_{\mathrm{T}}=1.3 \mathrm{~T}$ is $\mathrm{f}_{\mathrm{GW}} \approx 1.2$.

The density limit in H-plasmas with vertical target configurations is about 15-18\% lower than the H-mode DL pulses with outer strike point (OSP) on the horizontal target (Fig.8b). One explanation for this could be attributed to the different SOL profiles in these configurations, what can be explained by different neutral recycling patterns. It has to be noted that large differences between target profiles in divertor configurations with strike points on horizontal (HT) and vertical tiles (VT) were observed earlier in different machines and also attributed to different neutral recycling patterns: in VT configurations, recycling of the neutrals from the target had a larger probability to be ionized on flux surfaces hitting the target near the strike point, compared to HT configurations, which appeared to be more open to neutrals (see e.g. review paper [22] and [23]). Presumably due to steeper radial pressure profiles and correspondingly due to larger pressure gradients in the SOL in pulses with VT configurations, the ballooning stability threshold is exceeded at smaller electron densities than in pulses with HT configurations, leading to the reduced H-mode DL.

Another, maybe more straightfoward explanation could derived from simply the fact that the triangularity was $20-25 \%$ higher in the HT configurations which assumingly leads to a larger achievable radial pressure gradient in the edge region due to more stabilised MHD activity [24]. Contrarily, in discharges in VT configurations with lower triangularity compared to the investigated HT discharges, the ballooning stability threshold is supposedly exceeded at a lower electron density leading to the reduced H-mode DL. The impact of fuelling on MHD stability (broadening effect of pressure profile) is currently under investigation [25, 26, 27].

At ASDEX Upgrade, however, the H-mode density limit shows an increase with heating power [21]. The possible explanation of the different dependence on the $P_{\text {heat }}$ could be that the fuelling efficiency by gas puff is reduced at high densities and only a small fraction of the fuelled gas reaches the confined region. In comparison to the gas puff, the fuelling rate by the AUG NBIs $\left(\approx 10^{21} \mathrm{D} / \mathrm{s}\right.$ versus $10^{22} \mathrm{D} / \mathrm{s}$ by gas puffing $)$ is relatively large and it is not negligible because the 
major part of the injected $\mathrm{D}$ atoms are delivered directly into the confined region. This may explain the increase of $f_{G W}$ with the increase of the NBI heating power on AUG. On the JET machine, the puff rate $\left(\approx 10^{23} \mathrm{D} / \mathrm{s}\right)$ is two orders of magnitude larger than the rate of the injected neutral D-atoms $\left(\approx 10^{21} \mathrm{D} / \mathrm{s}\right)$ and the fuelling by NBI is thus negligible.

We should note that the opacity for neutrals crossing the separatrix of JET and AUG SOLs could be different. The JET SOL may be more opaque for neutrals compared to AUG and the fraction of recycling or puffed neutrals crossing the separatrix may be small. To clarify this issue further, H-mode density limit experiments with only RF wave heating (ICRH) power are required.

\section{Impact of the plasma shape on the density limit}

The impact of the plasma shape on the H-mode density limit has been analysed in the experiments by varying magnetic equilibria at constant neutral beam auxiliary heating NBIpower of 12MW. Fig. 9 illustrates the behaviour of the inner divertor BeII emission as well as of Greenwald fractions, $\mathrm{f}_{\mathrm{GW}}$, for a H-mode DL gas ramp-up discharges in JET-ILW with Low triangularity pulses, $\mathrm{B}_{\mathrm{T}} / \mathrm{I}_{\mathrm{p}}=1.8 \mathrm{~T} / 1.5 \mathrm{MA}, \mathrm{q}_{95}=3.6$

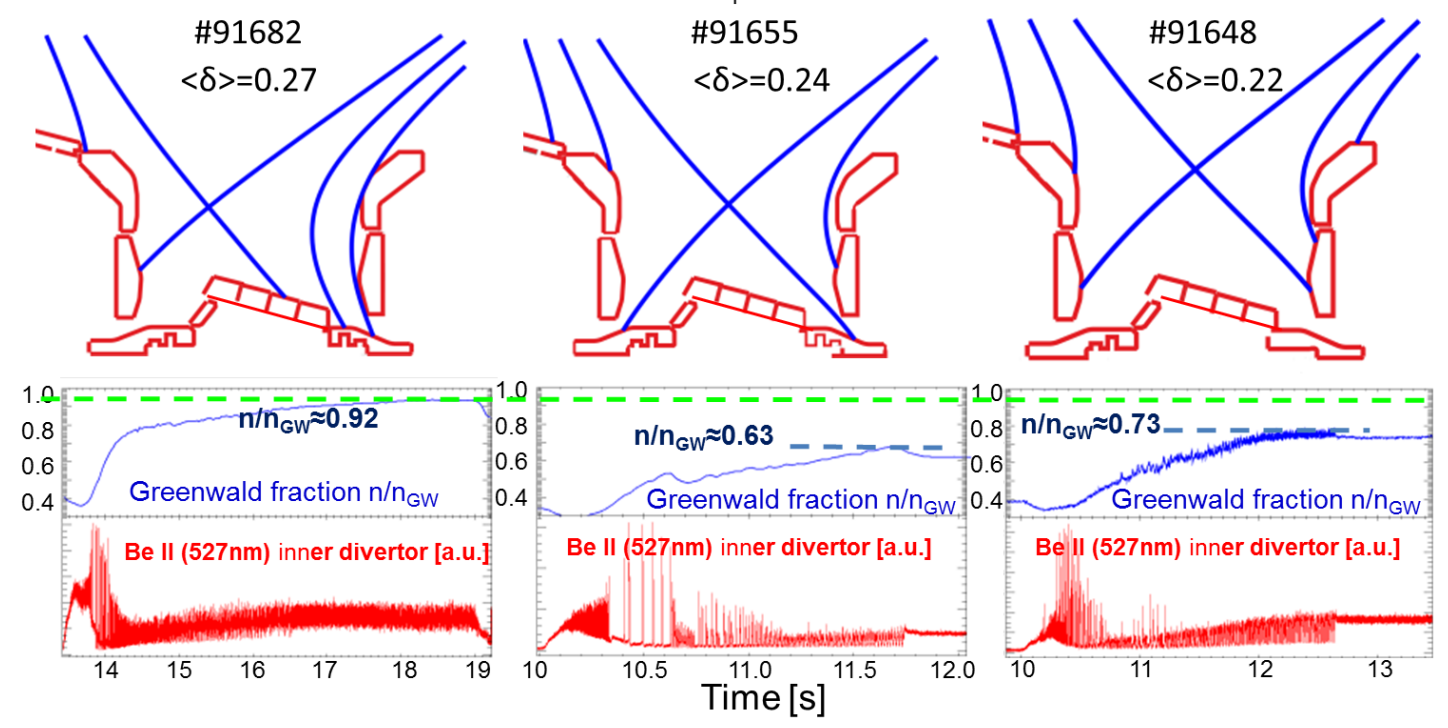

Fig.9 Impact of magnetic shapes on the H-mode density limit in low $\delta$ plasma pulses.

different plasma shapes at constant main plasma parameters $\mathrm{B}_{\mathrm{T}} / \mathrm{I}_{\mathrm{p}}=1.8 \mathrm{~T} / 1.5 \mathrm{MA}, \mathrm{q}_{95}=3.6$.

The plasma triangularity in these pulses was low and varied from $\delta=0.22$ to $\delta=0.27$. The plasma pulse when the effective neutral recycling is reduced, by placing both strike points close to the pumping duct (the so-called 'corner' divertor configuration) shows a strong reduction of the 
H-mode density limit. The Greenwald fraction reached in discharge with corner configuration is $\mathrm{f}_{\mathrm{GW}}=0.63$ versus 0.73 and 0.93 for the other shown plasma shape discharges. In the corner configuration with the strike points close to the cryopump the pumping efficiency of the recycled particles is increased, impacting the SOL profiles. We speculate that the enhanced pumping efficiency of the recycled particles leads to steeper radial pressure profiles and, correspondingly, to larger pressure gradients in the SOL. The ballooning stability threshold is thus exceeded at smaller electron densities in corner configurations than in pulses with noncorner configurations leading to the reduced H-mode DL. Reduction of the $\mathrm{f}_{\mathrm{GW}}$ supports the idea that the density limit addresses the SOL physics.

The physics mechanism resulting in lower $f_{G W}$ in plasma discharges in corner configuration is not yet understood and is under investigation.

\section{Comparison with the theoretical model}

In [5], the author, R. J. Goldston, suggests that the H-Mode density limit may be caused by an MHD instability in the SOL close to the separatrix rather than originating in the core plasma or pedestal. This idea has been triggered by observations showing that the ballooning parameter $\alpha \equiv-R q^{2} \frac{\partial \beta}{\partial r}$, derived from the heat flux width, almost linearly increases with $f_{G W}$ until detachment occurs. Here $q$ is the safety factor and $\beta$ is the total plasma beta (the ratio of plasma pressure to magnetic pressure). The ballooning $\alpha$ parameter was derived [5] under the assumption that the pressure gradient scale length at the separatrix is approximately equal to the experimentally measured $\lambda_{\mathrm{q}}\left(\alpha \equiv-R q^{2} \beta / \lambda_{q}\right)$. It is expected to scale similarly, but we should mention here that the pressure scale length should still be broader than $\lambda_{q}$ since $\lambda_{T}=7 / 2 \lambda_{q}$ and $\lambda_{n}$ is longer than $\lambda_{T}[28]$.

Assuming that the SOL $\beta$ limit is defined by criticality to MHD instability, characterized as $\alpha_{\text {crit }} \sim C_{\alpha}\left(1+\kappa^{2}\right)^{\gamma}$, the Goldston model gives

$f_{G W, \text { Goldston }}=8.13 \cdot C_{\alpha} \frac{\bar{n}}{n_{\text {sep }}}\left(\frac{q_{c y l} R B}{a} P_{S O L}\right)^{-1 / 8}\left(1+\kappa^{2}\right)^{\gamma-3 / 2}\left[\frac{2 \bar{A}}{(1+\bar{Z})}\right]^{9 / 16}\left(\frac{Z_{\text {eff }}+4}{5}\right)^{-1 / 8}$ where $\mathrm{q}_{\mathrm{cyl}}$ is the cylindrical approximation for the safety factor, $\kappa$ is the plasma elongation, $a$ and $R$ the horizontal minor and major radiuses, $\mathrm{P}_{\mathrm{SOL}}$ is the power which crosses from the core region into the SOL, $B$ is the total magnetic field strength, $\bar{n}$ and $n_{\text {sep }}$ are the line-averaged and separatrix densities, $Z_{\text {eff }}$ and $\bar{Z}$ are the "effective charge" and "average charge" of all ions. 
Generally speaking, $\mathrm{f}_{\mathrm{GW}}$ shows little variation with key parameters. On the contrary, it shows a relatively strong dependence on the average atomic mass, $\bar{A}$, of the plasma species.

For elliptic plasmas with elongation $\kappa$, it could be adapted according to Ref. [29]

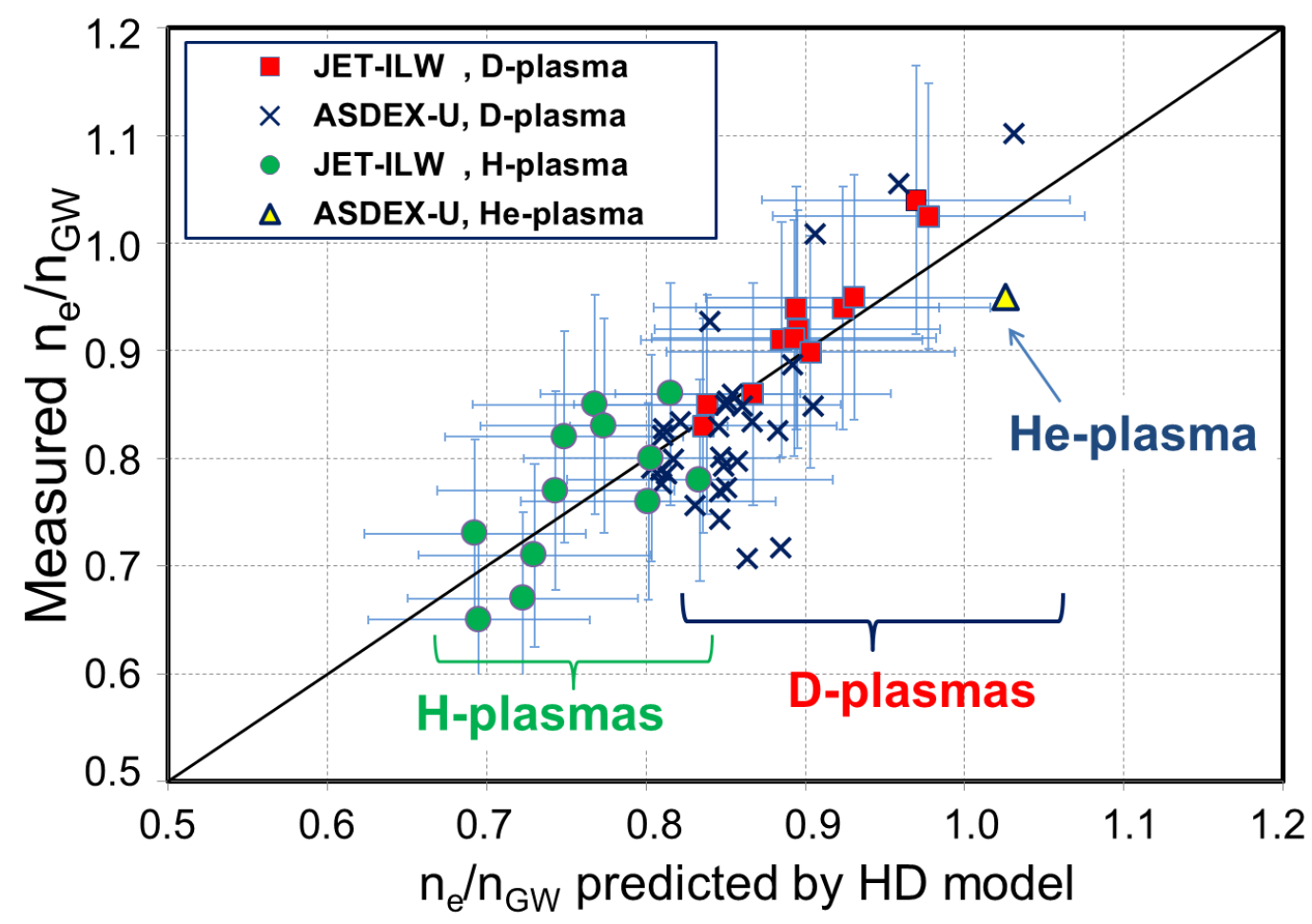

Fig. 10 Experimental measurements of the Greenwald fraction, $\mathrm{n}_{\mathrm{e}} / \mathrm{n}_{\mathrm{GW}}$, in gas fuelled H-Mode DL discharges in JET-ILW and AUG tokamaks, plotted against the Heuristic Drift (HD) model [5]. Filled circle symbols represent the result in H-plasmas on JET-ILW.

$\alpha_{\text {crit }} \approx 0.4 s\left(1+\kappa^{2}\right)$, where $s=d \ln q / d \ln r$ is the magnetic shear (typically $s=2$ ).

Experimental measurements of the H-mode density limit expressed in terms of the Greenwald fraction, $\mathrm{f}_{\mathrm{GW}}$, in both tokamaks, JET-ILW and AUG, are plotted against the $\mathrm{f}_{\mathrm{GW}}$ derived from the Goldston prediction (see Fig.10). The experimental results contain only data sets of the D-plasmas and H-plasmas (filled circle symbols) which correspond to plasma configurations with strike points on the vertical targets. Error bars on the graph represent the statistical error. Additionally the figure contains the result of H-mode density limit experiment in He-plasma achieved on ASDEX Upgrade. The Goldston model gives a good agreement with recent measurements concerning the dependence on main plasma parameters. Also the model predicts a strong dependence on the average atomic mass, $\bar{A}$, of the plasma species, $f_{G W} \propto \bar{A}^{9 / 16}$, which is confirmed experimentally on JET-ILW in D- and H-plasmas.

The ballooning instability triggers the SOL turbulence. In the previous investigation [30], it was shown that the turbulence overshooting throughout the pedestal leads to a non-vanishing turbulent 
transport within the barrier and provides a coupling of core and SOL turbulence despite the transport barrier. Eventually, it leads to the H-L transition.

\section{Extrapolation to ITER}

Based on the above findings, it is possible to attempt a prediction of the density limit on ITER. The design values for ITER the baseline 15MA, Q=10 inductive $\mathrm{H}$-mode burning plasma discharges [1] are as follows: $\mathrm{R}=6.2 \mathrm{~m}, \mathrm{a}=2.0 \mathrm{~m}, \kappa=1.7, \mathrm{~B}_{\mathrm{T}}=5.3 \mathrm{~T}, \mathrm{I}_{\mathrm{p}}=15 \mathrm{MA}, \mathrm{q}_{\text {cyl }}=2.42, \mathrm{Z}_{\mathrm{eff}}=1.6$, $\bar{n} / n_{\text {sep }}=3$. We assuming that ITER will operate in deuterium-tritium (D-T) mixtures using $50 \%$ of $\mathrm{D}$ and $50 \%$ of $\mathrm{T}$ and nominal operation of ITER with $\mathrm{Q}=10$ corresponds to $\mathrm{P}_{\mathrm{SOL}}=100 \mathrm{MW}$. Inserting the above into the $\mathrm{f}_{\mathrm{GW}}$ scaling predicted by Goldston, we find the estimate $\mathrm{f}_{\mathrm{GW}} \approx 1.03$. Note that ITER will operate with impurity seeding and that the scaling should be confirmed in dedicated experiments with impurity seeding.

We can conclude that the JET and ITER operational domains are significantly broadened when increasing the plasma effective mass (e.g. tritium or deuterium-tritium operation): the density limit for the L-mode back transition is increased. At the same time, the $\mathrm{L}$ to $\mathrm{H}$ power threshold is reduced [31].

\section{Conclusion}

To investigate the physics mechanisms that lead to $\mathrm{H}$-mode density limit and correspondingly to the H-L back transition, a series of experiments was started at JET in D(with plasma currents $\mathrm{I}_{\mathrm{p}}=1.75-2.5 \mathrm{MA}$ and toroidal magnetic fields $\mathrm{B}_{\mathrm{T}} \approx 1.8-3.4 \mathrm{~T}$ ) and in $\mathrm{H}$ plasmas $\left(\mathrm{I}_{\mathrm{p}}=0.9-2.0 \mathrm{MA}\right.$ and $\left.\mathrm{B}_{\mathrm{T}} \approx 1.3-1.8 \mathrm{~T}\right)$ at varying input power levels in the low triangularity vertical target configuration with high deuterium/protium fuelling. The observed $\mathrm{H}$-mode density limit on JET in deuterium as well as in hydrogen plasmas demonstrates similar operation phases. These phases are distinguished by their impact on the plasma density and stored energy. They are identified as

$\checkmark$ stable H-mode where the plasma density increases at a stable confinement,

$\checkmark$ dithering cycling phase during which could be distributed in two sub-phases:

- the early 'dithering phase' with confinement degradation and

- the late phase with the breakdown of the H-mode.

$\checkmark$ L-mode where the density decreases over the full profile and the temperature further reduces. 
During a stable H-mode the plasma density increases and the pedestal temperature decreases at constant pressure and stable confinement. During the earlier dithering phase the density still increases marginally with a drop in $\mathrm{T}_{\mathrm{e} \text {,ped }}$ and a reduction in the pedestal pressure due to reduction of the confinement. The late dithers demonstrate strong altering of the stored energy as well as a density drop by $15 \%$ which finally leads to the back transition into the L-mode.

We found that the density limit is not related to an inward collapse of the hot discharge core induced by overcooling of the plasma periphery by radiation. Indeed, independently of the isotopic effect, the total radiated power $\left(P_{\text {rad, total }}\right)$ as well as the radiated power in the main chamber $\left(P_{\text {rad, bulk }}\right)$ stay almost constant during the H-mode phase until the H-L transition.

The experimental study of the H-mode density limit in H-plasmas supports the view of the earlier observation on JET in D-plasmas [4,12] that detachment or the X-point MARFE itself do not trigger the H-L transition and thus do not present a limit on the plasma density. Independently of the isotopic mass of the main plasma, it has been observed that the transition from H-mode to L-mode is not always an abrupt event but may exhibit a series of H-L-H transitions (“dithering H-mode"), or a gradual transition (which is orders of magnitude longer than the energy confinement time $\tau_{\mathrm{E}}$ ).

Although the operation phases are identical for D- and H-plasmas, the DL shows a strong dependence on the isotopic mass, the DL is up to $35 \%$ lower in the H-plasma than in the deuterium plasma. Basically, the density limit in H mode on JET-ILW is nearly independent of the power in the range of observed heating powers both in D-plasmas and in H-plasmas.

On the other hand, the DL shows a strong reduction of $f_{\mathrm{GW}}$ in the corner configuration, supporting the idea that the density limit may originate in the scrape off layer (SOL) physics.

The measured Greenwald fractions in the vertical targets configuration are found to be consistent with the predictions from a theoretical model which suggests that the H-Mode density limit may be caused by MHD-driven turbulence in the SOL close to the separatrix, rather than originating in the core plasma or pedestal. Ballooning instability triggers the SOL turbulence which is spreading into the transport barrier and finally leads to the coupling of core and SOL transport throughout the pedestal [30] and correspondingly leads to H-L transition. When applying this model validated on JET-ILW to ITER, the H-mode density limit is estimated to be close to the Greenwald density in ITER D-T operation.

To summarise the JET results on the H-mode formation and back transition to L-mode at high density, we conclude that the JET and ITER operational domains are significantly 
broadened when increasing the plasma effective mass (e.g. tritium or deuterium-tritium operation), i.e. the $\mathrm{L}$ to $\mathrm{H}$ power threshold is reduced whereas the density limit for the L-mode back transition is increased.

\section{Acknowledgement}

This work has been carried out within the framework of the EUROfusion Consortium and has received funding from the Euratom research and training programme 2014-2018 under grant agreement No 633053. The views and opinions expressed herein do not necessarily reflect those of the European Commission. 


\section{References}

[1] Progress in the ITER Physics Basis, Edd., 2007 Nucl. Fusion 47 S1 sqq

[2] Zohm H. et al 2013 Nucl. Fusion 53073019

[3] Greenwald M. et al 2002 Plasma Phys. Control. Fusion 44 R27

[4] Huber A. et al 2016 Nucl. Mater. Energy in press

[5] Goldston R. J. et al 2015 J. Nucl. Mater. 463 397-400

[6] Borras K. et al 1998 Contrib. Plasma Phys. 38 130-135

[7] Huber A. et al 2015 J. Nucl. Mater. 463 445-449

[8] Brezinsek S. et al 2013 J. Nucl. Mater. 438 S303-S308

[9] de la Luna E. et al 2004 Rev. Sci. Instrum. 753831

[10] Tokar M. Z. 2009 Phys. of Plasmas 16020704

[11] Chankin A. V. and Saibene G. 1999 Plasma Phys. Control. Fusion 41 913-930

[12] Huber A. et al 2013 J. Nucl. Mater. 438 S139-S147

[13] Borras K. et al 2004 Nucl. Fusion 44752

[14] Huber A et al 2012, Rev. Sci. Instrum. 83 10D511

[15] Golub G.H. and Van Loan C.F. 1983 John Hopkins University Press, Baltimore

[16] Fenstermacher M. E. et al 1997 Rev. Sci. Instrum. 68974

[17] Huber A et al 2003 J. Nucl. Mater. 313-316 925

[18] Summers H.P. Atomic Data and Analysis Structure, URL: http://www.adas.ac.uk/

[19] Fujimoto K. et al 2009 Plasma and Fusion Res. 4025

[20] Loarte A et al 1998 Nucl. Fusion 38331

[21] Bernert M. et al 2015 Plasma Phys. Control. Fusion 57014038 (12pp)

[22] Loarte A. 2001 Plasma Phys. Control. Fusion 43 R183-R224

[23] Wiesen S. et al 2016 Proc. 26th IAEA Fusion Energy Conf. (Kyoto, Japan, 2016) EX/P66

[24] Maggi C.F. et al 2015 Nuclear Fusion 55113031

[25] Saarelma S.et al 2013 Nucl. Fusion 53123012

[26] Pamela S. et al 2016 Plasma Phys. Control. Fusion 58014026

[27] Pamela S. et al 2016 Proc. 26th IAEA Fusion Energy Conf. (Kyoto, Japan,2016)

[28] Sun H. J et al 2015 Plasma Phys. Control. Fusion 57125011

[29] Bateman G. et al 2003 Phys. Plasmas 104358

[30] Ghendrih Ph. et al 2007 J. Nucl. Mater. 363-365 581-585 
[31] The JET Team (presented by K Thomsen) 1999 Plasma Phys. Control. Fusion 41 A617A624 